\title{
A GROSS MEASURE PROPERTY
}

BY

\section{LAWRENCE R. ERNST}

\begin{abstract}
We prove that there exists a subset $E$ of $[0,1] \times \mathbf{R}^{2}$ such that the 2-dimensional Gross measure of $E$ is 0 , while the 1-dimensional Gross measure of $\{z:(y, z) \in E\}$ is positive for all $y \in[0,1]$. It is known that for Hausdorff measures no set exists satisfying these conditions.
\end{abstract}

1. Introduction. A special case of $[1,2.10 .25,2.10 .27]$ states that for any positive integers $k, m, n$ there exists $c \in \mathbf{R}$ such that

$$
\int^{*} \mathcal{H}^{k}\{z:(y, z) \in A\} d \mathscr{L}^{m} y \leqslant c \mathcal{F C}^{k+m}(A)
$$

for all $A \subset \mathbf{R}^{m} \times \mathbf{R}^{n}$, where $\mathcal{F}^{m}, \mathfrak{L}^{m}$ denote $m$-dimensional Hausdorff and Lebesgue measure respectively. It immediately follows that the same type of relation holds with the Hausdorff measures replaced by the spherical, $\mathcal{T}$, Carathéodory or Gillespie measures (provided $k<n$ ), since the ratios between the Hausdorff measure and any one of these other measures of the same dimension are bounded $[1,2.10 .6]$. It is also known that the inequality holds with $c=1$ in the case of spherical or Gillespie measures [1, 2.10.27, 3.2.45], but not in the case of Hausdorff measures [2].

In this paper we establish that no such relation is true for Gross measures (denoted $\mathcal{G}$ ) by constructing a subset $E$ of $[0,1] \times \mathbf{R}^{2}$ for which $\mathcal{G}^{2}(E)=0$ (Theorem 4.1), while $\mathcal{S}^{1}\{z:(y, z) \in E\}=2$ for all $y \in[0,1]$ (Lemma 3.1). The method of proof that $\mathcal{G}^{2}(E)=0$ uses the structure theory of $[1,3.3]$ and, in particular, incorporates some of the ideas of $[1,3.3 .19]$.

One consequence of our result is that some theorems concerning $\left(\mathrm{SC}^{m}, m\right)$ rectifiable sets $[1,3.2 .14]$ do not hold for $\left(\mathcal{G}^{m}, m\right)$ rectifiable sets. For example our set $E$ shows that $[1,3.2 .22]$ is no longer true if $\mathcal{H C}$ is replaced by $\mathcal{G}$.

The author wishes to thank the referee for his corrections and useful suggestions.

2. Preliminaries. In general we adopt the notation and terminology of [1]. Presented in this section are some additional definitions that we use.

Throughout this paper, unless otherwise restricted, $0<y<1$, while $n \geqslant 3$

Received by the editors August 10, 1976 and, in revised form, February 21, 1977.

AMS (MOS) subject classifications (1970). Primary 28A35, 28 A75.

Key words and phrases. Gross measure, Hausdorff measure, structure theory, $\left(\mathcal{G}^{m}, m\right)$ rectifiable. 
is an integer. We also note that we do not distinguish between $\mathbf{R}^{2}$ and $\mathbf{C}$ or between $\mathbf{R} \times \mathbf{R}^{2}$ and $\mathbf{R}^{3}$.

For $a, b \in \mathbf{R}^{m}$ let $[a, b]$ denote the closed line segment with endpoints $a, b$.

Define $\lambda: \mathbf{R} \rightarrow \mathbf{C}, \xi: \mathbf{R} \times \mathbf{C} \rightarrow \mathbf{R} \times \mathbf{C}, \lambda(x)=\exp (x \mathbf{i}), \xi(x, z)=(x, z \lambda(x))$ for $x \in \mathbf{R}, z \in \mathbf{C}$.

The following series of definitions culminate in the definition of the set $E$ referred to in the Introduction. For each closed circular disk $S=\mathbf{B}(a, r) \subset$ $\mathbf{R}^{2}$ and positive integer $i<n$, let

$$
\begin{aligned}
x_{i, n}(S) & =\inf \{x:(x, w) \in S\}+(2 i-1) r / n, \\
w_{i, n}(S) & = \begin{cases}\sup \left\{w: \mathbf{B}\left[\left(x_{i, n}, w\right), r / n\right] \subset S\right\} & \text { if } i \text { is odd, } \\
\inf \left\{w: \mathbf{B}\left[\left(x_{i, n}, w\right), r / n\right] \subset S\right\} & \text { if } i \text { is even, }\end{cases} \\
F_{n}(S) & =\left\{\mathbf{B}\left[\left(x_{j, n}, w_{j, n}\right), r / n\right]: j=1, \ldots, n\right\} .
\end{aligned}
$$

Then inductively define families $G_{3}, G_{4}, G_{5}, \ldots$, of closed circular disks by taking $G_{3}=\{\mathbf{B}(0,1)\}, G_{n+1}=\bigcup\left\{F_{n+1}(S): S \in G_{n}\right\}$ for $n \geqslant 3$. Finally let $A=\bigcap_{n=3}^{\infty} \cup G_{n}$ and $E=\xi([0,1] \times A)$.

Define $p(x, z)=x, q(x, z)=z$ for $(x, z) \in \mathbf{R} \times \mathbf{R}^{2}$.

For $x \in \mathbf{R}^{m} \sim\{0\}$ let $\tau(x)=x /|x|$.

For $w \in \mathbf{R}$ define $\rho_{w} \in \mathbf{O}^{*}(3,1), \rho_{w}(x)=q(x) \cdot \lambda(w)$ for $x \in \mathbf{R}^{3}$. (Note that throughout this paper a "." between two complex numbers denotes an inner product, not complex multiplication.)

Take $r_{n}=6 / n !$. Note that diam $S=2 r_{n}$ for $S \in G_{n}$.

Let $A_{y}=E \cap p^{-1}[y], G_{n y}=\left\{\xi(\{y\} \times S): S \in G_{n}\right\}, \quad K_{n}=\{\xi([(j-$ 1) $\left.\left.\left.r_{n}, j r_{n}\right] \times S\right): j=1,2, \ldots, r_{n}^{-1}, S \in G_{n}\right\}$.

3. Some lemmas. In 3.1-3.5 we prove a few lemmas about the Hausdorff and Gross measures of $E$ and certain of its subsets. In the remainder of this section the key results are Lemmas 3.10,3.13, 3.15, each of which is used in Theorem 4.1 to show that a different subset of $E$ has $\mathcal{G}^{2}$ measure 0 .

3.1. Lemma. $\mathcal{G}^{1}\left(E_{y}\right)=\mathcal{H}^{1}\left(E_{y}\right)=2$.

Proof. Since $\mathcal{L}^{1}\left[\rho_{y}\left(E_{y}\right)\right]=2$ it follows from $[1,2.10 .8]$ that $\mathcal{G}^{1}\left(E_{y}\right)>2$. Furthermore, since $G_{n y}$ covers $E_{y}$ and $\Sigma_{S \in G_{n y}} \operatorname{diam} S=2$ we have $\mathcal{H}^{\prime}\left(E_{y}\right)<$ 2. Finally we recall that $\mathcal{G}^{1} \leqslant \mathscr{H}^{1}[1,2.10 .6]$.

3.2. Lemma. If $S \in G_{n, y}$ then $\mathcal{G}^{1}\left(S \cap E_{y}\right)=\mathfrak{H}^{1}\left(S \cap E_{y}\right)=2 r_{n}$.

Proof. This lemma follows by applying the method used to establish Lemma 3.1.

3.3. Lemma. $\mathcal{H}^{2}(E)<\infty$. 
Proof. We observe that $K_{n}$ covers $E$, card $K_{n}=r_{n}^{-2}$, and that diam $p(S)$ $=r_{n}$, diam $q(S) \leqslant 3 r_{n}$ for every $S \in K_{n}$. Consequently

$$
(\pi / 4) \sum_{s \in K_{n}}(\operatorname{diam} S)^{2} \leqslant 5 \pi / 2
$$

Hence $\mathcal{T C}^{2}(E) \leqslant 5 \pi / 2$.

3.4. Definition. For $w \in \mathbf{R}$ let $I(w)=E \cap\{x: q(x) \wedge \lambda(w)=0\}$.

3.5. LEMMA. $\mathcal{H}^{2}[I(w)]=0$ for all $w \in \mathbf{R}$.

Proof. The result follows from Lemma 3.3 and the fact that if $x \in\left[0, \frac{1}{2}\right]$ then

$$
\begin{aligned}
& \mathcal{H}^{2}\left[I(w) \cap p^{-1}\left(\left[0, \frac{1}{2}\right]\right)\right]=\mathcal{H}^{2}\left[I(w+x) \cap p^{-1}\left(\left[x, x+\frac{1}{2}\right]\right)\right] \\
& \mathcal{H}^{2}\left[I(w) \cap p^{-1}\left(\left[\frac{1}{2}, 1\right]\right)\right]=\mathcal{H}^{2}\left[I(w-x) \cap p^{-1}\left(\left[\frac{1}{2}-x, 1-x\right]\right)\right] .
\end{aligned}
$$

3.6. Definitions. For $\varnothing \neq T \subset S \in G_{n y}$ let $\beta_{n}(T)=S$ and $c_{n}(T)$ denote the center of $S$; for $x \in S \sim\left\{c_{n}(S)\right\}$ define $\eta_{n}(x)=\tau\left(q\left[x-c_{n}(S)\right]\right)$.

Let $\Delta_{n y}$ denote the set of all closed proper line segments $\left[a_{1}, a_{2}\right] \in p^{-1}\{y\}$ for which there exists $S \in G_{n y}$ satisfying $\left\{q\left(a_{1}\right), q\left(a_{2}\right)\right\} \subset \operatorname{Bdry} q(S), c_{n}(S)$ $\notin\left[a_{1}, a_{2}\right]$.

Let $L \in \Delta_{n, y}$ define

$$
\begin{aligned}
& \alpha(L)=\inf \left\{\left|\eta_{n}(x) \wedge \lambda(y)\right|: x \in L\right\}, \\
& R(L)=\beta_{n}(L) \cap\left\{x:\left[c_{n}\{x\}, x\right] \cap L \neq \varnothing\right\}, \\
& M(L)=G_{n+1, y} \cap\left\{T: T \subset \beta_{n}(L), \eta_{n}(T) \subset \eta_{n}(L)\right\}, \\
& m(L)=\operatorname{card} M(L) .
\end{aligned}
$$

3.7. LEMMA. If $L \in \Delta_{n, y}, \alpha(L) \neq 0$ and $n \geqslant 2^{4}[\alpha(L)]^{-1}(\operatorname{diam} L)^{-1} r_{n}$, then

$$
m(L) /(n+1) \geqslant 2^{-3} \alpha(L)(\operatorname{diam} L) r_{n}^{-1} \text {. }
$$

Proof. Since $\operatorname{card}\left[G_{n+1, y} \cap\left\{T: T \subset \beta_{n}(L), \rho_{y}(T) \cap \rho_{y}(L) \neq \varnothing\right\}\right]<$ $2 m(L)+3$ and $r_{n+1}=r_{n} /(n+1)$, it follows

$$
[2 m(L)+3] 2 r_{n} /(n+1)>\operatorname{diam} \rho_{y}(L) \text {. }
$$

Furthermore, we note that if $x, w$ are the endpoints of $L$ and $\alpha(L)=\mid \eta_{n}(x)$ $\wedge \lambda(y) \mid$, then

$$
\begin{aligned}
\operatorname{diam} \rho_{y}(L) & =|\tau[q(w-x)] \cdot \lambda(y)| \operatorname{diam} L \geqslant\left|\left[\mathbf{i} \eta_{n}(x)\right] \cdot \lambda(y)\right| \operatorname{diam} L \\
& =\alpha(L) \operatorname{diam} L .
\end{aligned}
$$

We then combine these last two results with the given bound on $n$ to obtain our conclusion. 
3.8. Lemma. If $L \in \Delta_{n, y}$ and $n \geqslant 2^{10}(\operatorname{diam} L)^{-2} r_{n}^{2}$, then $m(L) /(n+1) \geqslant$ $2^{-9}(\operatorname{diam} L)^{2} r_{n}^{-2}$.

PRoof. Consider $a \in R(L) \cap q^{-1}\left(\operatorname{Bdry} q\left[\beta_{n}(L)\right]\right)$ satisfying

$$
\left|\eta_{n}(a) \wedge \lambda(y)\right|=\sup \left\{\left|\eta_{n}(x) \wedge \lambda(y)\right|: x \in L\right\} .
$$

Then choose $L_{1} \in \Delta_{n y}$ with $a \in L_{1} \subset R(L)$ and diam $L_{1}=(\operatorname{diam} L) / 8$. We observe that if $w$ is either of the two points of $\beta_{n}(L)$ satisfying $\mid \rho_{y}(w-$ $\left.c_{n}\{w\}\right) \mid=r_{n}$, then $|a-w| \geqslant(\operatorname{diam} L) / 2$; consequently $\left|\eta_{n}(a) \wedge \lambda(y)\right| \geqslant$ $(\operatorname{diam} L) /\left(4 r_{n}\right)$. We combine this with the fact that $\left|\eta_{n}(a) \wedge \eta_{n}(x)\right| \leqslant$ (diam $L) /\left(8 r_{n}\right)$ for all $x \in L_{1}$, to obtain $\alpha\left(L_{1}\right) \geqslant(\operatorname{diam} L) /\left(8 r_{n}\right)$. We then apply Lemma 3.7 to $L_{1}$.

3.9. Definition. Let $F_{1}$ denote the set of all points $x$ of $E$ for which $\left\{\eta_{n}(x)\right.$ : $n \geqslant 3\}$ is not dense in $\mathbf{S}^{1}$.

3.10. LEMMA. $\mathcal{H}^{2}\left(F_{1}\right)=0$.

Proof. Consider any closed proper subarc $J$ of $\mathbf{S}^{1}$. We will obtain our result by showing that

$$
\mathcal{H}^{2}\left[E \cap \bigcap_{n=3}^{\infty}\left\{x: \eta_{n}(x) \notin J\right\}\right]=0 .
$$

To do this we let $J_{1}$ denote the closed subarc of $\mathbf{S}^{1}$ with the same midpoint as $J$, satisfying $\mathcal{H}^{1}\left(J_{1}\right)=\mathcal{H}^{1}(J) / 2$. Choose an integer $\nu>2^{10}\left(\operatorname{diam} J_{1}\right)^{-2}$ for which $r_{v} \leqslant \mathcal{I C}^{1}(J) / 4$. Then inductively define the three sequences $B_{v}, B_{v+1}$, $B_{\nu+2}, \ldots, D_{\nu+1}, D_{\nu+2}, D_{\nu+3}, \ldots, C_{\nu+1}, C_{\nu+2}, C_{\nu+3}, \ldots$, by taking $B_{\nu}=K_{\nu}$, and for $n \geqslant \nu$ letting

$$
\begin{aligned}
& D_{n+1}=K_{n+1} \cap\left\{T: T \subset \cup B_{n}\right\}, \\
& B_{n+1}=D_{n+1} \cap\left\{T: \eta_{n}(T) \cap\left(\mathbf{S}^{1} \sim J\right) \neq \varnothing\right\}, \\
& C_{n+1}=D_{n+1} \cap\left\{T: \eta_{n}\left[T \cap p^{-1}\{\sup p(T)\}\right] \subset J_{1}\right\} .
\end{aligned}
$$

We observe that if $x \in T \in C_{n+1}$ and $t=\sup p(T)$, then $(t, q(x) \lambda[t-$ $p(x)]) \in T \cap p^{-1}\{t\}$ and $\left|\eta_{n}[(t, q(x) \lambda[t-p(x)])] \wedge \eta_{n}(x)\right|=\sin [t-p(x)]$ $\leqslant \sin r_{n+1} \leqslant \sin \left[\mathcal{T C}^{1}(J) / 4\right]$; consequently $B_{n+1} \cap C_{n+1}=\varnothing$. Furthermore, if $S \in B_{n}, w \in\left\{\sup p(T): T \in D_{n+1}, T \subset S\right\}$ and $L$ is the line segment satisfying the conditions $L \in \Delta_{n, w}, \beta_{n}(L)=p^{-1}\{w\} \cap S, \eta_{n}(L)=J_{1}$, then from Lemma 3.8 it follows that

$$
m(L) /(n+1) \geqslant 2^{-9}(\operatorname{diam} L)^{2} r_{n}^{-2}=2^{-9}\left(\operatorname{diam} J_{1}\right)^{2} .
$$

Therefore, either $B_{n}=\varnothing$ or 


$$
\begin{aligned}
\mathcal{H}^{2}(E & \left.\cap \bigcup B_{n+1}\right) / \mathcal{H}^{2}\left(E \cap \bigcup B_{n}\right)=\left(\operatorname{card} B_{n+1}\right) /\left(\operatorname{card} D_{n+1}\right) \\
\leqslant 1-\left(\operatorname{card} C_{n+1}\right) /\left(\operatorname{card} D_{n+1}\right) & \leqslant 1-2^{-9}\left(\operatorname{diam} J_{1}\right)^{2} .
\end{aligned}
$$

Thus $\mathcal{H}^{2}\left[\cap_{n=\nu}^{\infty}\left(E \cap \cup B_{n}\right)\right]=0$, from which (1) follows.

3.11. Corollary. $\mathcal{H}^{1}\left(F_{1} \cap E_{y}\right)=0$.

Proof. If $t, w \in[0,1]$ then

$$
F_{1} \cap E_{w}=\left\{(w, q(x) \lambda(w-t)): x \in F_{1} \cap E_{t}\right\} ;
$$

consequently $\mathcal{H}^{1}\left(F_{1} \cap E_{w}\right)=\mathcal{H}^{1}\left(F_{1} \cap E_{t}\right)$. Furthermore, by $[1,2.10 .27]$ and Lemma 3.10 we have

$$
\int_{0}^{1} \mathcal{K}^{1}\left(F_{1} \cap E_{y}\right) d \mathfrak{L}^{1} y \leqslant(4 / \pi) \mathcal{H}^{2}\left(F_{1}\right)=0 .
$$

3.12. Definimions. For $a \in \mathbf{R}^{3}, 0<r<r^{\prime} \leqslant \infty, V \in \mathbf{G}(3,1), 0<s<1$, let

$$
\begin{aligned}
\mathbf{X}(a, r, V, s) & =\mathbf{R}^{3} \cap\left\{x: s^{-1} \operatorname{dist}(x-a, V)<|x-a|<r\right\}, \\
\mathbf{Y}\left(a, r, r^{\prime}, V, s\right) & =\operatorname{Clos}\left[\mathbf{X}\left(a, r^{\prime}, V, s\right) \sim \mathbf{X}(a, r, V, s)\right] .
\end{aligned}
$$

3.13. Lemma. If $a \in E_{y} \sim F_{1}, V \in \mathbf{G}(3,1), V \subset p^{-1}\{0\}, v \in \mathbf{S}^{1}, \mathbf{R} v=$ $q(V), b=|v \cdot \lambda(y)|>0$ and $0<s<b$, then

$$
\begin{gathered}
\limsup _{n \rightarrow \infty} \operatorname{card}\left[G_{n+1, y} \cap\left\{T: T \subset \beta_{n}\{a\} \cap \mathbf{Y}\left(a, r_{n} s / 8, r_{n} s, V, s\right)\right\}\right] /(n+1) \\
\geqslant 2^{-7} b s .
\end{gathered}
$$

PRoof. Let $V_{n}=\{0\} \times\left(\mathbf{R}\left[\eta_{n}(a) \mathrm{i}\right]\right), W_{n}=\mathbf{Y}\left(a, r_{n} s / 4, r_{n} s / 2, V_{n}, s / 2\right)$. Let $N$ denote the set of all integers $n$ such that $n \geqslant \sup \left\{64 / s^{2}, 2^{8} /(b s)\right\}, \mid v \wedge$ $\left[\eta_{n}(a) \mathrm{i}\right] \mid<s / 4$ and $\left\{x: \operatorname{dist}\left(x, W_{n}\right) \leqslant 2 r_{n+1}\right\} \subset \mathrm{Y}\left(a, r_{n} s / 8, r_{n} s, V, s\right)$. We note that $\sup N=\infty$ since $a \notin F_{1}$; consequently it suffices to obtain our result with $\lim \sup _{n \rightarrow \infty}$ replaced by $\inf _{n \in N}$. To do this we choose $n \in N$ and consider $a_{1}, \quad a_{2}, \quad a_{3} \in p^{-1}\{y\} \cap q^{-1}\left[\operatorname{Bdry} q\left(\beta_{n}\{a\}\right)\right] \cap\{x: q(x-a)$. $\left[\eta_{n}(a)\right.$ i $\left.] \geqslant 0\right\}$ satisfying $\left|a_{1}-a\right|=r_{n} s / 2,\left|a_{2}-a\right|=r_{n} s / 4, q\left(a_{3}-a\right) \cdot \eta_{n}(a)$ $=0$. Let $L=\left[a_{1}, a_{2}\right]$. We note that $\cup M(L) \subset\left\{x: \operatorname{dist}[x, R(L)]<2 r_{n+1}\right\}$. Therefore to complete the proof we need only show that $R(L) \subset W_{n}$ and $m(L) /(n+1) \geqslant 2^{-7} b s$.

To obtain the former relation we simply observe that

$$
\left|\eta_{n}(a) \wedge \eta_{n}(x)\right| \leqslant\left|\eta_{n}(a) \wedge \eta_{n}\left(a_{1}\right)\right| \leqslant s / 2 \text { for every } x \in R(L),
$$
while since $n \geqslant 64 / s^{2}$ we also have

$$
\begin{aligned}
\left|a_{3}-a\right| & =\left(r_{n}^{2}-\left|a-c_{n}\{a\}\right|^{2}\right)^{1 / 2} \leqslant\left(1-[1-2 /(n+1)]^{2}\right)^{1 / 2} r_{n} \\
& \leqslant 2 r_{n} /(n+1)^{1 / 2} \leqslant r_{n} s / 4 .
\end{aligned}
$$


To compute $m(L)$ we first note that diam $L \geqslant r_{n} s / 4$. To obtain a lower bound on $\alpha(L)$ we let $\delta_{1}=\sin ^{-1}\left[\left|\eta_{n}(a) \wedge \lambda(y)\right|\right], \delta_{2}=\sin ^{-1}[|(v i) \wedge \lambda(y)|]$, $\delta_{3}=\sin ^{-1}\left[\left|(v i) \wedge \eta_{n}(a)\right|\right]$. Then since $\delta_{1} \geqslant\left|\delta_{2}-\delta_{3}\right|$ it follows that

$$
\begin{aligned}
\left|\eta_{n}(a) \wedge \lambda(y)\right| & =\sin \left(\delta_{1}\right) \geqslant\left|\sin \left(\delta_{2}\right)-\sin \left(\delta_{3}\right)\right| \\
& =||(v \mathbf{i}) \wedge \lambda(y)|-|(v \mathbf{i}) \wedge \eta_{n}(a)|| \\
& =|| v \cdot \lambda(y)|-| v \wedge\left[\eta_{n}(a) \mathbf{i}\right]|| \geqslant b-s / 4 \geqslant 3 b / 4,
\end{aligned}
$$

which we combine with the inequalities (2) and $s<b$ to find that $\alpha(L) \geqslant$ $b / 4$. Finally we see that Lemma 3.7 is applicable since $n \geqslant 2^{8} /(b s)$.

3.14. LEMMA. $E_{y}$ is purely $\left(\mathcal{H C}^{1}, 1\right)$ unrectifiable.

Proof. It follows from Corollary 3.11 and Lemma 3.13 that

$$
\lim _{s \rightarrow 0^{+}} \limsup _{n \rightarrow \infty} \mathcal{H}^{1}\left[E_{y} \cap \mathbf{X}\left(a, r_{n} s, \operatorname{ker} \rho_{y+\pi / 4}, s\right)\right] r_{n}^{-1} s^{-2}=\infty
$$

for $\mathcal{H}^{1}$-almost all $a$ in $E_{y}$; consequently $\mathcal{L}^{1}\left[\rho_{y+\pi / 4}\left(E_{y}\right)\right]=0$ by $[1,3.3 .9]$. Similarly $\mathcal{L}^{1}\left[\rho_{y+3 \pi / 4}\left(E_{y}\right)\right]=0$. Hence $E_{y}$ is purely $\left(\mathcal{H}^{1}, 1\right)$ unrectifiable by $[1,3.2 .27]$.

3.15. Lemma. $E$ is purely $\left(\mathcal{H}^{2}, 2\right)$ unrectifiable.

Proof. If $W$ is an $\left(\mathcal{H}^{2}, 2\right)$ rectifiable Borel subset of $E$, then it follows from $[1,3.2 .29]$ that $\mathcal{F}^{2}$-almost all of $W$ is contained in the union of some countable family of 2 dimensional submanifolds of class 1 of $\mathbf{R}^{3}$. Let $B$ denote a member of such a family and let $M=B \cap W$. We will complete the proof by showing that $\mathcal{H}^{2}(M)=0$.

To do this we first observe that for $\mathcal{L}^{1}$-almost all $y$ in $[0,1]$ we have by $[1,3.2 .22(2)]$ that $M \cap p^{-1}\{y\}$ is $\left(\mathcal{H}^{1}, 1\right)$ rectifiable and hence $\mathcal{H C}^{1}(M \cap$ $\left.p^{-1}\{y\}\right)=0$ by Lemma 3.14. It then follows from [1, 3.2.22(3)] that

$$
\int_{M} a p J_{1} p d \mathcal{H}^{2}=\int_{0}^{1} \mathcal{H}^{1}\left(M \cap p^{-1}\{y\}\right) d \mathcal{L}^{1} y=0 .
$$

Consequently, $a p J_{1} p(x)=0$ for $\mathcal{H}^{2}$-almost all $x$ in $M$, which combined with $[1,3.2 .19]$ implies that $\operatorname{Tan}^{2}\left(\mathcal{T C}^{2} L M, b\right)=p^{-1}\{0\}$ for $\mathcal{T}^{2}$-almost all $b$ in $M$. We next choose any $b \in M$ for which $\operatorname{Tan}^{2}\left(\mathcal{H C}^{2} L M, b\right)=p^{-1}\{0\}$ and observe that by $[1,3.1 .19(4)]$ there exists a neighborhood $T$ of $b$ in $\mathbf{R}^{3}$ such that $q \mid(B \cap T)$ is univalent, $q(B \cap T)$ is convex, $\psi$ is of class 1 and $D \psi[q(b)]=$ $q^{*}$, where $\psi[q \mid(B \cap T)]^{-1}$. From the conditions on $\psi$ we find that there exists a convex neighborhood $S$ of $q(b)$ in $\mathbf{R}^{2}$ such that $\left\|D \psi(z)-q^{*}\right\|<\frac{1}{2}$ for all $z \in S$; consequently $\operatorname{Lip}\left[\left(\psi-q^{*}\right) \mid S\right]<\frac{1}{2}$, which in turn implies that $\operatorname{Lip}[(p \circ \psi) \mid S]<\frac{1}{2}$. We let $Z=\psi(S) \cap M$ and note that to finish the proof it suffices to show that $\mathcal{H}^{1}(Z)<\infty$.

To accomplish this we define $h: Z \rightarrow E_{0}, h(x)=(0, q(x) \lambda[-p(x)])$. Then 
since $\operatorname{Lip}[(p \circ \psi) \mid S]<\frac{1}{2}$ we see that for $x, w \in Z$,

$$
\begin{aligned}
|h(x)-h(w)| & \geqslant|q(x)-q(w)|-|p(x)-p(w)| \\
& \geqslant\left(\frac{1}{2}\right)|q(x)-q(w)| \geqslant\left(5^{1 / 2} / 4\right)|x-w| .
\end{aligned}
$$

Consequently $\operatorname{Lip}\left(h^{-1}\right) \leqslant 4 / 5^{1 / 2}$, which we combine with $[1,2.10 .11]$ and Lemma 3.1 to conclude that

$$
\mathcal{H}^{1}(Z) \leqslant \operatorname{Lip}\left(h^{-1}\right) \mathcal{H}^{1}[h(Z)] \leqslant\left(4 / 5^{1 / 2}\right) \mathcal{H}^{1}\left(E_{0}\right)<\infty .
$$

4. Principal theorem. We prove here that $\mathcal{G}^{2}(E)=0$. This result and Lemma 3.1 establish the claim made in the Introduction.

4.1. THEOREM. $\mathcal{G}^{2}(E)=0$.

Proof. Consider any $\theta \in \mathbf{O}^{*}(3,2)$. We will obtain our result by showing that $\mathbf{L}^{2}[\theta(E)]=0$. To do this we first choose $v \in \mathbf{S}^{2}$ satisfying $\mathbf{R} v=\operatorname{ker} \boldsymbol{\theta}$. If $q(v) \neq 0$ we then take any $\varepsilon>0$ and apply Lemma 3.5 to obtain a closed proper subarc $J$ of $\mathbf{S}^{1}$ whose midpoint is $\tau[\mathbf{i} q(v)]$ and which satisfies $\mathcal{H}^{2}[\cup\{I(w): \lambda(w) \in J\}]<\varepsilon$; we then let $F_{2}=\bigcup\{I(w): \lambda(w) \in J\}$. On the other hand, if $q(v)=0$ we take $F_{2}=\varnothing$. For $z \in q\left(E \sim F_{2}\right)$ define $\sigma(z) \in \mathbf{S}^{2}$, $\gamma(z) \in \mathbf{R} \times \mathbf{R}^{2}, g(z) \in[0, \pi]$, by $\sigma(z)=\tau[(1, \mathbf{i} z)], \gamma(z)=(0, q(v)-\mathbf{i} p(v) z)$ and $g(z)=|\arg (\mathbf{i} q[\gamma(z)])|$. Let $H=q\left(E \sim F_{2}\right) \cap\{z: g(z) \leqslant 1\}$, and define $f: H \rightarrow \mathbf{R} \times \mathbf{R}^{2}, f(z)=(g(z), z)$ for $z \in H$. Finally let

$$
K=E \sim\left[F_{1} \cup F_{2} \cup f(H) \cup p^{-1}\{0,1\}\right] .
$$

We next show that to complete the proof we need only establish that

$$
\mathfrak{L}^{2}[\theta(K)]=0 \text {. }
$$

For it follows from the definition of $\gamma, g$ and $f$ that $f$ is Lipschitzian, which together with Lemma 3.15 implies $\mathcal{H}^{2}[E \cap f(H)]=0$. Recalling Lemmas 3.1, 3.10 and the definition of $F_{2}$, we would then have $\mathcal{H}^{2}(E \sim K)<\varepsilon$, which together with $[1,2.10 .8,2.10 .6]$ would yield

$$
\mathcal{L}^{2}[\theta(E \sim K)] \leqslant \mathcal{G}^{2}(E \sim K) \leqslant \mathcal{H}^{2}(E \sim K)<\varepsilon .
$$

Finally this last result and (3) imply $\mathcal{L}^{2}[\theta(E)]=0$.

To obtain (3) we consider any $a \in K$, let $b=|\tau[q(\gamma[q(a)])] \cdot \lambda[p(a)]|$, and note that $b>0$ since $a \notin f(H)$. We will show that

$$
\lim _{s \rightarrow 0^{+}} \limsup _{n \rightarrow \infty} \mathcal{H}^{2}\left[E \cap \mathbf{X}\left(a, r_{n} s, \operatorname{ker} \theta, s\right)\right] r_{n}^{-2} s^{-4}=\infty \text {. }
$$

We then immediately have (3) by $[1,3.3 .9]$.

To deduce (4) we first choose $\nu_{1}, \nu_{2} \in \mathbf{S}^{2}$ so that $v, \nu_{1}, \nu_{2}$ is an orthonormal basis for $\mathbf{R}^{3}$ and $\sigma[q(a)]$ is a linear combination of $v$ and $\nu_{1}$. Let $P=\{a+d v$ $\left.+t \nu_{2}: d, t \in \mathbf{R}\right\}, V=\mathbf{R} \gamma[q(a)]$. For $x \in \mathbf{R}^{3}$ define 


$$
\Omega(x)=x-\left(\left[(x-a) \cdot \nu_{1}\right] /\left[\sigma[q(a)] \cdot \nu_{1}\right]\right) \sigma[q(a)] .
$$

We note that $\lim \Omega \subset P$ since $[\Omega(x)-a] \cdot \nu_{1}=0$ for all $x \in \mathbf{R}^{3}$. We also observe that since $\gamma[q(a)]=v-p(v)\left(1+[q(a)]^{2}\right)^{1 / 2} \sigma[q(a)]$ is a linear combination of $v$ and $\nu_{1}$, it follows that $[\Omega(a+x \gamma[q(a)])-a] \cdot \nu_{2}=0$ for all $x \in \mathbf{R}$ and hence

$$
\Omega(a+V) \subset a+\operatorname{ker} \theta .
$$

Let $k=\inf \left\{\left|\sigma[q(a)] \wedge \tau\left(x_{1}-x_{2}\right)\right|: x_{1}, x_{2} \in P, x_{1} \neq x_{2}\right\}$. We note that since $a \notin F_{2}$ it follows that $\sigma[q(a)] \notin \mathbf{R} v$ and consequently $k \neq 0$. We then choose $s$ satisfying $0<s<\inf \{4 b / k$, dist $[p(a),\{0,1\}]\}$ and let

$$
\begin{aligned}
& T_{n}=p^{-1}\{p(a)\} \cap \mathrm{Y}\left(a, k r_{n} s / 32, k r_{n} s / 4, V, k s / 4\right), \\
& Y_{n}=P \cap \mathbf{Y}\left(a, k r_{n} s / 64, r_{n} s / 4, \operatorname{ker} \theta, s / 2\right) .
\end{aligned}
$$

We next show that $\Omega\left(T_{n}\right) \subset Y_{n}$. To do this we consider any $x, w \in$ $p^{-1}\{p(a)\}, x \neq w$. Then since $|p(\sigma[q(a)])| \geqslant 2^{-1 / 2}>\frac{1}{2}$ we have

$$
|x-w| / 2 \leqslant|\sigma[q(a)] \wedge(x-w)| \leqslant|x-w|,
$$

while the definition of $k$ yields

$$
k|\Omega(x)-\Omega(w)| \leqslant|\sigma[q(a)] \wedge[\Omega(x)-\Omega(w)]| \leqslant|\Omega(x)-\Omega(w)| .
$$

From these inequalities and the relation $|\sigma[q(a)] \wedge(x-w)|=\mid \sigma[q(a)] \wedge$ $[\Omega(x)-\Omega(w)] \mid$ we obtain $\frac{1}{2} \leqslant|\Omega(x)-\Omega(w)| /|x-w| \leqslant 1 / k$, which we then combine with (5) to conclude $\Omega\left(T_{n}\right) \subset Y_{n}$.

Let

$$
Z_{n}=\left\{c_{n+1}(S): S \in G_{n+1, p(a)}, S \subset \beta_{n}\{a\} \cap T_{n}\right\}
$$

For $x \in Z_{n}$ define

$$
\begin{aligned}
\Gamma(x) & =(p[\Omega(x)], q(x) \lambda(p[\Omega(x)-x])) \\
Q_{n}(x) & =\left\{(y, z \lambda(y-p[\Gamma(x)])):|y-p[\Gamma(x)]|<2^{-8} k r_{n} s^{2}\right. \\
& \left.\quad z \in q\left(\beta_{n+1}[\Gamma(x)]\right)\right\} .
\end{aligned}
$$

Applying [1, 2.10.27], Lemma 3.2, and also noting that $p\left[Q_{n}(x)\right] \subset[0,1]$ because of the choice of $s$, we deduce that

$$
\mathcal{H}^{2}\left[E \cap Q_{n}(x)\right] \geqslant(\pi / 4) \mathcal{L}^{1}\left(p\left[Q_{n}(x)\right]\right) 2 r_{n+1}=2^{-8} \pi k r_{n}^{2} s^{2} /(n+1)
$$

for all $x \in Z_{n}$. Furthermore from Lemma 3.13, with $s$ replaced by $k s / 4$, it follows that

$$
\limsup _{n \rightarrow \infty}\left(\operatorname{card} Z_{n}\right) /(n+1) \geqslant 2^{-9} k b s .
$$

We then combine these last two results to obtain 


$$
\limsup _{n \rightarrow \infty} \mathcal{T C}^{2}\left[E \cap \bigcup_{x \in Z_{n}} Q_{n}(x)\right] r_{n}^{-2} \geqslant 2^{-17} \pi k^{2} b s^{3} .
$$

Consequently to complete the proof of (4) we need only establish that for $n$ sufficiently large,

$$
\bigcup_{x \in Z_{n}} Q_{n}(x) \subset \mathbf{X}\left(a, r_{n} s, \operatorname{ker} \theta, s\right) .
$$

To obtain (6) we choose $x \in Z_{n}$ and observe that since $\Omega(x) \in Y_{n}$, we have

$$
\operatorname{dist}\left[\Omega(x), \mathbf{R}^{3} \sim \mathbf{X}\left(a, r_{n} s, \operatorname{ker} \theta, s\right)\right] \geqslant|\Omega(x)-a| s / 2 \geqslant 2^{-7} k r_{n} s^{2} .
$$

Furthermore, it follows from the definition of $Q_{n}(x)$ that if $w \in Q_{n}(x)$ then $|p[w-\Gamma(x)]| \leqslant 2^{-8} k r_{n} s^{2},|q[w-\Gamma(x)]| \leqslant r_{n+1}+2^{-8} k r_{n} s^{2}$. We then combine these last three inequalities to deduce that to conclude (6) it suffices to show

$$
\lim _{n \rightarrow \infty}\left[\sup \left\{|\Omega(x)-\Gamma(x)|: x \in Z_{n}\right\} / r_{n}\right]=0 .
$$

To prove (7) we consider any $n \geqslant 5$ and $x \in Z_{n}$. We then let $h=p[\Omega(x)-$ $x], w_{1}=q[\Omega(x)-x], w_{2}=q[\Gamma(x)-x], u=q[x+\Gamma(x)] / 2$. Since $\Omega(x) \in$ $Y_{n}$ we see that $|h| \leqslant r_{n} s / 4 \leqslant r_{n}$. We also note that $\left|w_{1}\right|=|q(a)| \cdot|h| \leqslant r_{n}$, $\left|w_{2}\right|=2|q(x)| \sin (|h| / 2) \leqslant r_{n},|q(x-a)| \leqslant k r_{n} s / 4 \leqslant r_{n}$. Then since

$$
|u-q(a)| \leqslant\left|w_{2}\right| / 2+|q(x-a)| \leqslant 3 r_{n} / 2,
$$

$|q(a)| \geqslant \frac{1}{2}$ and $n \geqslant 5$, it follows that

$$
\tau[q(a)] \cdot \tau(u) \geqslant\left(1-9 r_{n}^{2}\right)^{1 / 2} \geqslant 1-r_{n} .
$$

Furthermore, if $h>0$ then $\tau\left(w_{1}\right)=\tau[q(a) \mathbf{i}]$ and $\tau\left(w_{2}\right)=\tau(u i)$, while if $h<0$ then $\tau\left(w_{1}\right)=\tau[q(a)(-\mathbf{i})]$ and $\tau\left(w_{2}\right)=\tau[u(-\mathbf{i})]$; consequently in either case $\tau\left(w_{1}\right) \cdot \tau\left(w_{2}\right) \geqslant 1-r_{n}$. Finally we conclude (7) by using this last inequality to compute

$$
\begin{aligned}
|\Omega(x)-\Gamma(x)|^{2} & =\left|w_{1}-w_{2}\right|^{2}=\left|w_{1}\right|^{2}+\left|w_{2}\right|^{2}-2\left|w_{1}\right| \cdot\left|w_{2}\right| \tau\left(w_{1}\right) \cdot \tau\left(w_{2}\right) \\
& \leqslant\left(\left|w_{1}\right|-\left|w_{2}\right|\right)^{2}+2\left|w_{1}\right| \cdot\left|w_{2}\right| r_{n} \leqslant\left(\left|w_{1}\right|-\left|w_{2}\right|\right)^{2}+2 r_{n}^{3},
\end{aligned}
$$

and then combining this result with the relation

$$
\begin{aligned}
|| w_{1}|-| w_{2}|| & =|| q(a)|\cdot| h|-2| q(x)|\sin (|h| / 2)| \\
& \leqslant\left(|q(x)|+r_{n}\right)|h|-2|q(x)| \sin (|h| / 2) \\
& \leqslant|h|+r_{n}|h|-2 \sin (|h| / 2) \\
& \leqslant r_{n}+r_{n}^{2}-2 \sin \left(r_{n} / 2\right) .
\end{aligned}
$$

\section{REFERENCES}

1. H. Federer, Geometric measure theory, Springer-Verlag, Heidelberg and New York, 1969. 
2. D. J. Ward, A counterexample in area theory, Proc. Cambridge Philos. Soc. 60 (1964), 821-845.

3. H. Whitney, Geometric integration theory, Princeton Univ. Press, Princeton, N. J., 1957.

Department of Mathematics, Queens College of the City Universty of New York, FLushing, NeW YoRK 11367

Current address: Research Center for Measurement Methods, Bureau of the Census, Washington, D. C. 20233 